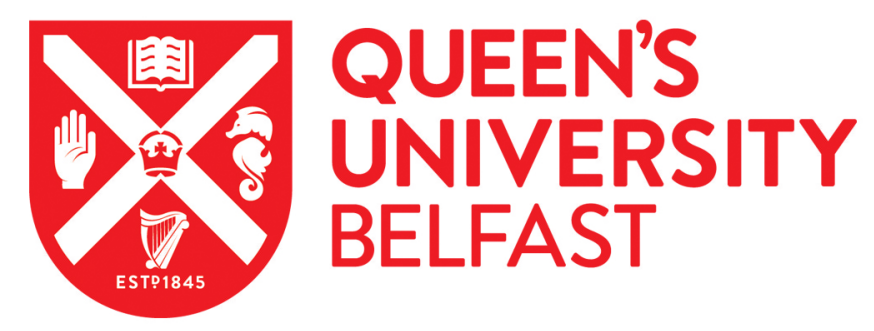

\title{
Nanoprecipitation preparation of low temperature-sensitive magnetoliposomes
}

Cheung, C. C. L., Monaco, I., Kostevšek, N., \& Al-Jamal, W. T. (2020). Nanoprecipitation preparation of low temperature-sensitive magnetoliposomes. COLLOIDS AND SURFACES B-BIOINTERFACES, 111453. https://doi.org/10.1016/j.colsurfb.2020.111453

Published in:

COLLOIDS AND SURFACES B-BIOINTERFACES

Document Version:

Peer reviewed version

Queen's University Belfast - Research Portal:

Link to publication record in Queen's University Belfast Research Portal

\section{Publisher rights}

Copyright 2020 Elsevier.

This manuscript is distributed under a Creative Commons Attribution-NonCommercial-NoDerivs License

(https://creativecommons.org/licenses/by-nc-nd/4.0/), which permits distribution and reproduction for non-commercial purposes, provided the author and source are cited.

\section{General rights}

Copyright for the publications made accessible via the Queen's University Belfast Research Portal is retained by the author(s) and / or other copyright owners and it is a condition of accessing these publications that users recognise and abide by the legal requirements associated with these rights.

Take down policy

The Research Portal is Queen's institutional repository that provides access to Queen's research output. Every effort has been made to ensure that content in the Research Portal does not infringe any person's rights, or applicable UK laws. If you discover content in the Research Portal that you believe breaches copyright or violates any law, please contact openaccess@qub.ac.uk. 


\section{Nanoprecipitation preparation of low temperature- sensitive magnetoliposomes}

5 Calvin C.L. Cheung ${ }^{a}$, Ilaria Monaco ${ }^{b}$, Nina Kostevšek ${ }^{c}$, Mauro Comes Franchini ${ }^{b}$, and

Wafa T. Al-Jamal ${ }^{*}$

a School of Pharmacy, Queen's University Belfast, Belfast, United Kingdom

${ }^{\mathrm{b}}$ Department of Industrial Chemistry "Toso Montanari", University of Bologna, Italy

${ }^{c}$ Department for Nanostructured Materials, Jožef Stefan Institute, Ljubljana, Slovenia

10

*Corresponding author:

Dr Wafa' T. Al-Jamal

School of Pharmacy

Queen's University Belfast

15 Belfast, BT9 7BL

United Kingdom

E-mail:w.al-jamal@qub.ac.uk 


\section{Abstract}

20 Lysolipid-containing thermosensitive liposomes (LTSL) have gained attention for triggered release of chemotherapeutics. Superparamagnetic iron oxide nanoparticles (SPION) offers multimodal imaging and hyperthermia therapy opportunities as a promising theranostic agent. Combining LTSL with SPION may further enhance their performance and functionality of LTSL. However, a major challenge in clinical translation of nanomedicine is the poor scalability and complexity of their preparation process. Exploiting the nature of self-assembly, nanoprecipitation is a simple and scalable technique for preparing liposomes. Herein, we developed a novel SPION-incorporated lysolipid-containing thermosensitive liposome (mLTSL10) formulation using nanoprecipitation. The formulation and processing parameters were carefully designed to ensure high reproducibility and stability of mLTSL10. The effect of

30 solvent, aqueous-to-organic volume ratio, SPION concentration on the mLTSL10 size and dispersity was investigated. mLTSL10 were successfully prepared with a small size $(\sim 100 \mathrm{~nm})$, phase transition temperature at around $42{ }^{\circ} \mathrm{C}$, and high doxorubicin encapsulation efficiency. Indifferent from blank LTSL, we demonstrated that mLTSL10 combining the functionality of both LTSL and SPION can be successfully prepared using a scalable nanoprecipitation approach. 


\section{Introduction}

Lysolipid-containing thermosensitive liposomes (LTSL) have been developed as a promising solution to enhance tumor drug bioavailability, in contrast to conventional liposomal

40 formulations that rely on passive release [1]. LTSL comprises base phospholipid 1,2dipalmitoyl-sn-glycero-3-phosphocholine (DPPC), lysolipid 1-stearoyl-2-hydroxy-sn-glycero3-phosphocholine (MSPC), and PEG-lipid 1,2-distearoyl-sn-glycero-3-phosphoethanolamineN-[methoxy(polyethylene glycol)-2000] (DSPE-PEG2000). Lysolipids participate in the stabilization of membrane pores during phase transition, allowing burst release of entrapped hydrophilic drug; while PEG-lipids provide interfacial stabilizing (stealth) effect to prolong blood circulation and also facilitate drug release [2]. The most clinically advanced LTSL is ThermoDox®, a LTSL formulation optimized for the burst release of doxorubicin upon applying mild hyperthermia $\left(40-42{ }^{\circ} \mathrm{C}\right)$. To date, it is the first and only thermosensitive liposomal formulation to reach clinical trials, evaluating the treatment of solid tumors in combination with radiofrequency ablation and mild hyperthermia [3].

Additional modalities have been included in the LTSL platform to monitor and improve the performance of drug release. Metallic nanoparticles, such as superparamagnetic iron oxide nanoparticles (SPION) [4], gold nanoparticles [4,5] and copper sulfide nanoparticles [6], offer attractive optical and magnetic properties. They have been incorporated into LTSL as liposome-nanoparticle hybrids, for potential non-invasive bioimaging modality and trigger release controlled through external stimuli [4]. SPION-loaded liposomes, or magnetoliposomes, are particularly attractive for their promising multifunctionality. Magnetoliposomes enable magnetic targeted drug delivery [7,8], and act as an efficient MRI contrast agent with enhancing $\mathrm{T}_{2}$ contrast, for image-guided drug delivery applications [9].

60 Once they reached the targeted site, the embedded SPION can generate heat when exposed to external stimuli, such as near-infrared laser irradiation or alternative magnetic field, thus 
heating up lipid bilayer and trigger drug release $[10,11]$. Consequently, drug release can be controlled spatially and temporally. Moreover, magnetic hyperthermia treatment can trigger cell death by promoting apoptosis and necrosis or even via thermal ablation $[12,13]$.

65 Furthermore, the incorporation of SPION have been reported to stabilize the lipid bilayer and reduces unfavorable premature drug leakage from liposomes [14].

Despite the promising potentials of magnetoliposomes, their clinical translation has been slow; a significant hurdle in nanomedicines translation besides safety, is the complexity in scaling up laborious manufacturing processes. To date, most of the magnetoliposome formulations

70 utilized conventional batch methods, namely reverse phase evaporation or lipid film hydration, combined with sonication or extrusion. These are cumbersome bench-scale techniques, which are challenging to scale up and come along with other issues including low production yield, poor batch-to-batch reproducibility and cost ineffectiveness [15]. To date, reports on the scalable preparation of magnetoliposomes have been lacking. In this regard, nanoprecipitation is a scalable approach that enables the formation of liposomes in one-step via self-assembly, without the need to homogenize vesicles size [16,17]. Briefly, a water-miscible organic solution of lipids (and SPION) is mixed with an aqueous solution, where lipid molecules selfassemble into liposomes spontaneously as they become less soluble in the aqueous non-solvent phase. Liposome sizes are controlled simply by adjusting the formulation and processing 80 parameters, abolishing the need of downsizing and homogenization [18]. Owing to the nature of self-assembly, prepared liposomes have narrow size distribution and high reproducibility [19]. Moreover, nanoprecipitation of liposomes can be made continuous with the help of microfluidics. Microfluidics, the manipulation of fluid in microscale channels, have drawn much attention in recent years for the production of liposomes in a well-controlled, 85 reproducible and high-throughput manner [20]. 
We have shown previously the optimized preparation of LTSL and the entrapment of a hydrophobic drug using the microfluidics nanoprecipitation approach [21]. Herein, this work demonstrates for the first time the preparation of SPION-loaded LTSL (mLTSL) using nanoprecipitation. We have demonstrated the successful preparation and characterization of

90 homogeneous, nano-sized (100 and $200 \mathrm{~nm}$ ) SPION-loaded LTSL, with hydrophilic drug loading capability. 


\section{Materials and methods}

\subsection{Materials}

1,2-dipalmitoyl-sn-glycero-3-phosphocholine (DPPC) and 1,2-distearoyl-sn-glycero-3phosphoethanolamine-N-[methoxy(polyethylene glycol)-2000] (DSPE-PEG2000) were generous gifts from Lipoid GmbH (Ludwigshafen, Germany). 1-stearoyl-2-hydroxy-snglycero-3-phosphocholine (MSPC) was purchased from Avanti Polar Lipids (AL, US). Doxorubicin hydrochloride (DOX) was purchased from Apollo Scientific (Cheshire, UK).

100 Ammonium sulfate $\left(\left(\mathrm{NH}_{4}\right)_{2} \mathrm{SO}_{4}\right)$, ethanol, 4-(2-hydroxyethyl)-1-piperazineethanesulfonic acid (HEPES), sodium chloride $(\mathrm{NaCl})$, tetrahydrofuran, Triton ${ }^{\mathrm{TM}} \mathrm{X}-100$ were purchased from Sigma-Aldrich (Dorset, UK). Nitric acid for trace analysis was purchased from VWR (Leicestershire, UK).

\subsection{Preparation of N-palmitoyl-6-nitrodopamine-coated iron oxide nanoparticles}

105 Hydrophobic N-palmitoyl-6-nitrodopamine (PNDA)-coated superparamagnetic iron oxide nanoparticles (SPION) was synthesized and characterized as we reported previously [22]. Briefly, oleic acid (OA)-coated SPION was first synthesize by thermal decomposition of iron pentacarbonyl in octyl ether in the presence of oleic acid as described in the literature [23]. PNDA ligand was synthesized as described in the literature [24]. OA-coated SPION was ligand

110 exchanged to PNDA to obtain PNDA-coated SPION, where a solution of $20 \mathrm{mg} \mathrm{mL}^{-1}$ PNDA in THF/DMSO (2/1, v/v) was added dropwise to a solution of $3 \mathrm{mg} \mathrm{mL}^{-1}$ OA-coated SPION in THF. The mixture was stirred at $50{ }^{\circ} \mathrm{C}$ for $24 \mathrm{~h}$, then washed by THF and centrifuged for 1 $\mathrm{h}$ at $10000 \mathrm{~g}$ three times. The resultant PNDA-coated SPION (hereinafter called SPION) were dispersed in THF and stored at $4{ }^{\circ} \mathrm{C}$. The mean diameter of SPION was $4.5 \mathrm{~nm}$, characterized

115 by transmission electron microscopy analysis; PNDA-coating was characterized and confirmed 
by Fourier-transform infrared spectroscopy; magnetic properties of SPION was measured by vibrating-sample magnetometry and superparamagnetism was confirmed [22].

\subsection{Preparation of SPION-loaded LTSL (mLTSL10)}

A mixture of SPION and 10 mM LTSL10 (DPPC/MSPC/DSPE-PEG $2000,80 / 10 / 10$ molar ratio) $\mathrm{PEG}_{2000}(2.79 \mathrm{mg})$ and 0 - $400 \mu \mathrm{g}$ SPION, per mL of THF/ethanol (1/1, v/v; unless otherwise stated). Typically, $0.5 \mathrm{~mL}$ of the lipid mixture and $\left(\mathrm{NH}_{4}\right)_{2} \mathrm{SO}_{4}$ solution $\left(240 \mathrm{mM}\left(\mathrm{NH}_{4}\right)_{2} \mathrm{SO}_{4}\right.$, $\mathrm{pH}$ 5.4) were preheated to $60{ }^{\circ} \mathrm{C}$ in a water bath. The volume of $\left(\mathrm{NH}_{4}\right)_{2} \mathrm{SO}_{4}$ used depends on the designated aqueous-to-organic volume ratio. The lipid mixture was withdrawn and rapidly injected into the $\left(\mathrm{NH}_{4}\right)_{2} \mathrm{SO}_{4}$ solution, and mixed until viscous fingering was no longer visible. The formed liposomes were annealed at $60{ }^{\circ} \mathrm{C}$ for $1.5 \mathrm{~h}$. Annealed liposomes were purified and buffer exchanged to HEPES-buffered saline (HBS; 20 mM HEPES, 137 mM NaCl, pH 7.4) by size-exclusion chromatography (SEC) using Sephadex G-25 in PD-10 desalting column.

\subsection{Particle size distribution analysis by dynamic light scattering (DLS)}

130 Z-average diameter and dispersity were determined using Zetasizer Nano ZS90 (Malvern Panalytical, Worcestershire, UK) equipped with a $4.0 \mathrm{~mW}$ He-Ne laser operating at $633 \mathrm{~nm}$ with photodiode detector at a detection angle at $90^{\circ}$. Liposomal samples were diluted 10-fold in DW and transferred to low-volume polystyrene cuvettes. Z-average and dispersity of each sample were obtained as the mean of three measurements. The term "dispersity" is used instead 135 of "polydispersity index", in accordance with recommendations of IUPAC [25].

\subsection{Atomic absorption spectroscopy (AAS)}

Total concentrations of iron content of samples were determined by atomic absorption spectroscopy (AAS) using an Agilent 55 AA (Agilent, CA, US) equipped with Lumina hollow cathode lamp (PerkinElmer, UK). Iron absorbance was measured at absorption wavelength of 
$140248.33 \mathrm{~nm}$. The system was blanked by deionized water, calibrated standards were prepared by diluting an iron standard solution (Sigma, UK) to 1, 2 and $5 \mathrm{ppm}\left(\mu \mathrm{g} \mathrm{mL} \mathrm{m}^{-1}\right)$. Recalibration was performed between every ten samples. Samples were first completely dried off using a block heater, then $1 \mathrm{~mL}$ of concentrated nitric acid was added and heated at $80{ }^{\circ} \mathrm{C}$ for $2 \mathrm{~h}$ for acid digestion. Digested samples were diluted appropriately by deionized water and filtered

145 through a $0.45 \mu \mathrm{m}$ syringe filter before AAS measurement.

\subsection{Differential scanning calorimetry (DSC)}

Thermograms were recorded on TA Q200 DSC (TA Instruments, DE, US), in the temperature range of $35-50{ }^{\circ} \mathrm{C}$ with a heating rate of $1{ }^{\circ} \mathrm{C} \mathrm{min}^{-1}$. Purified mLTSL10 samples were concentrated by centrifugation to lipid concentration of $10 \mathrm{mM}$ lipid. $20 \mu \mathrm{L}$ of samples and HBS reference were transferred to two respective DSC pans and sealed with DSC hermetic lids (TA Instruments, DE, US).

\subsection{Transmission electron microscopy (TEM)}

TEM micrographs were imaged using a JEOL JEM-1400 Plus transmission electron microscope, operating at an accelerating voltage of $120 \mathrm{kV}$. Purified mLTSL10 samples were

155 diluted 100 times by deionized water; $2.5 \mu \mathrm{L}$ of sample was added on a carbon-coated 400 mesh copper grid (Ted Pella, Inc., Redding, CA, US) and allowed to air-dry for TEM imaging.

\subsection{DOX loading SPION-loaded LTSL using the pH-gradient remote loading method}

DOX was loaded into liposomes using a pH-gradient remote loading method. Following buffer exchange of the external buffer to HBS, the liposomes were incubated with DOX at DOX-to-

160 phospholipid molar ratio of $1: 20$ at $37^{\circ} \mathrm{C}$ for $1.5 \mathrm{~h}$. After incubation, liposomes were purified from unencapsulated DOX by SEC as described above. To quantify DOX encapsulation efficiency (DOX EE), liposomes before and after purification were diluted to the same lipid concentration and then solubilized by Triton X-100 to release encapsulated DOX. A final 
concentration of $0.1 \mathrm{v} / \mathrm{v} \%$ Triton X-100 was used, which corresponds to a phospholipid-todetergent molar ratio of 1:20, sufficient to ensure complete solubilization of liposomes [26]. DOX fluorescence intensity was measured using microplate reader with excitation and emission wavelength of $485 \mathrm{~nm}$ and $590 \mathrm{~nm}$, respectively. The concentration of DOX in the wells were within the linear region. DOX EE was then calculated by comparing the fluorescence intensity $(\mathrm{I}(\mathrm{t}))$ of the samples before and after purification:

$$
D O X E E=\frac{I(t) \text { after } \text { purification }}{I(t) \text { before purification }}
$$

\subsection{Statistical analysis}

Student's unpaired two-tailed t-test and one-way analysis of variance (ANOVA) followed by Fisher's least significant difference (LSD) test were used to assess statistical significance between group means [27,28]. All analyses were performed, with the significance level $\alpha$ of

175 0.05, using GraphPad Prism 7.0 (GraphPad Software Inc., CA, US). 


\section{Results}

\subsection{SPION precipitates in the presence of high-salt containing media and PEG-lipid}

To form SPION-loaded LTSL (mLTSL10) by nanoprecipitation, a water-miscible organic

180 phase is to be injected into the aqueous phase. Ethanol was used to dissolve the LTSL10 lipids (DPPC/MSPC/DSPE-PEG $2000,80 / 10 / 10$ molar ratio) [21]. Tetrahydrofuran (THF) is miscible with both water and ethanol, and able to solubilize SPION, therefore an ideal solvent for mLTSL10 preparation. To introduce SPION to the LTSL10 formulation, SPION could be added to either the lipid-containing ethanol phase or the aqueous phase. To investigate the

185 metastability and solubility of SPION when dispersed in these conditions, $200 \mu \mathrm{g} \mathrm{mL}^{-1}$ SPION in THF was dispersed in ethanol, DW, $\left(\mathrm{NH}_{4}\right)_{2} \mathrm{SO}_{4}$ solution and $\mathrm{HBS}$, with a final THF volume fraction of 1 v/v \% (Supplementary Figure S1a). Although SPION was insoluble in ethanol and aqueous solution alone, it was metastable when dispersed in THF/ethanol and THF/DW with overnight stability. However, in the presence of salt $\left(\left(\mathrm{NH}_{4}\right)_{2} \mathrm{SO}_{4}\right.$ and HBS), SPION

190 precipitated rapidly and completely settled overnight. While SPION can be dispersed in THF/DW, the use of DW as an aqueous phase for nanoprecipitation is not desirable due to the high dispersity of the resultant vesicles [18,29]. In fact, $\mathrm{HBS}$ and $\left(\mathrm{NH}_{4}\right)_{2} \mathrm{SO}_{4}$ were the preferred aqueous phase for their relevance with biological and drug loading applications. Therefore, SPION was dispersed in the organic phase of THF/ethanol along with the lipids. Hereinafter,

195 THF/ethanol was used as the organic phase and the volume fraction of THF in ethanol is implied by “\% THF”.

Subsequently, mLTSL10 mixture comprising $200 \mu \mathrm{g} \mathrm{mL}^{-1}$ SPION and $10 \mathrm{mM}$ LTSL10 lipid was dispersed in 1\% THF (Supplementary Figure S1b). Unexpectedly, the SPION rapidly precipitated in the presence of the lipids. To identify the cause, SPION was mixed with the 200 three lipid components of LTSL10 (8 mM DPPC, $1 \mathrm{mM}$ MSPC and $1 \mathrm{mM}$ DSPE-PEG 2000 ) 
respectively. Clearly, SPION remained metastable with DPPC and MSPC but was precipitated with DSPE-PEG 2000 . It was evident that the presence of DSPE-PEG 2000 disrupted the metastable SPION from the $1 \%$ THF solvent system. However, when SPION and DSPE$\mathrm{PEG}_{2000}$ were dispersed in THF alone, the mixture remained stable (Supplementary Figure

205 S1c). Therefore, it was hypothesized that the ratio between ethanol (to solubilize phospholipid) and THF (to solubilize SPION) could be optimized to achieve a stable dispersion of mLTSL10.

The stability of the mLTSL10 mixture in $0 \%$ to $100 \%$ THF was studied overnight (Figure 1). At $0 \%$ (absolute ethanol) to $40 \% \mathrm{THF}$, a decreasing amount of SPION was precipitated. At $60 \%$ and $80 \%$ THF, all components were soluble with overnight stability. In 100\% THF,

210 however, the mixture appeared turbid with the presence of precipitates. Therefore, the optimal volume fraction of THF should be greater than $40 \%$ and less than $100 \%$ THF.

\subsection{THF affects LTSL10 size and dispersity but not membrane integrity}

To determine the optimal THF volume fraction for mLTSL10 preparation, the effect of THF on the size and dispersity of LTSL10 was first investigated. The organic phase ( $0-75 \%$ THF)

215 containing $10 \mathrm{mM}$ LTSL10 was injected into the aqueous phase $\left(\left(\mathrm{NH}_{4}\right)_{2} \mathrm{SO}_{4}\right.$ solution $)$ at $60{ }^{\circ} \mathrm{C}$ with an aqueous-to-organic volume ratio (VR) of 3 (Figure 2a, Supplementary Figure S2a). One-way ANOVA was conducted to study the effect of \%THF on the size and the dispersity of LTSL10. There were statistically significant differences in both size $\left(F_{4,17}=162.2, p<0.001\right)$ and dispersity $\left(\mathrm{F}_{4,17}=4.934, \mathrm{p}=0.008\right)$. LTSL10 size increased somewhat exponentially with

$220 \mathrm{THF}$, from about $80-100 \mathrm{~nm}$ in $0-40 \% \mathrm{THF}$, to about $230 \mathrm{~nm}$ in $50 \%-60 \% \mathrm{THF}$, up to above $1000 \mathrm{~nm}$ in $75 \%$ THF. In contrast, LTSL10 dispersity increased moderately from 0.1 in $0-60 \%$ THF to 0.2 in $75 \%$ THF. Despite the variation in LTSL10 size with different THF volume fraction, LTSL10 remained uniform (dispersity $<0.2$ ). To validate the membrane integrity of the LTSL10 prepared in the presence of THF, samples were remotely loaded with 
225 DOX. Regardless of the \%THF used for preparation, LTSL10 have DOX encapsulation efficiency (DOX EE) above $80 \%(83.86 \pm 3.56 \% ; \mathrm{n}=4)$, indicating the presence of a transmembrane $\mathrm{pH}$-gradient (necessary for DOX loading) thus the integrity of the membrane.

\subsection{Uniform mLTSL10 successfully prepared with size dependent on injection volume ratio}

230 After successful validation of LTSL10, SPION was included to optimize the preparation of mLTSL10. The organic phase (20 - 75\% THF) containing $10 \mathrm{mM}$ LTSL10 and $300 \mu \mathrm{g} \mathrm{mL}^{-1}$ SPION (equivalent to SPION-to-lipid ratio of $30 \mu \mathrm{g} \mu \mathrm{mol}^{-1}$ [22]) was injected into the aqueous phase $\left(\left(\mathrm{NH}_{4}\right)_{2} \mathrm{SO}_{4}\right)$ at $60{ }^{\circ} \mathrm{C}$ with an aqueous-to-organic volume ratio of 3 (Figure $\mathbf{2 b}$, Supplementary Figure S2b). Expectedly, in $20-40 \%$ THF, due to the low stability of the mLTSL10 mixture, liposome size distributions were multimodal and highly dispersed (>0.4); moreover, SPION precipitates could be observed. Therefore, one-way ANOVA was conducted to study the effect of \%THF on the size and the dispersity of mLTSL10 prepared only in between $50-75 \%$ THF. There were statistically significant differences in both size $\left(\mathrm{F}_{2,10}=\right.$ 164.3, $\mathrm{p}<0.001)$ and dispersity $\left(\mathrm{F}_{2,10}=12.67, \mathrm{p}=0.002\right)$. Both the liposome size and dispersity

rose significantly with increasing THF volume fraction, from 50\% THF (Z-average $=194.4$ $\mathrm{nm}$; dispersity $=0.107)$ to $60 \%$ THF $\left(Z\right.$-average $=325.2 \mathrm{~nm}, \mathrm{t}_{10}=3.947, \mathrm{p}=0.003$; dispersity $\left.=0.182, \mathrm{t}_{10}=2.348, \mathrm{p}=0.041\right)$ and $75 \%$ THF $\left(\mathrm{Z}\right.$-average $=708.7 \mathrm{~nm}, \mathrm{t}_{10}=18.12, \mathrm{p}<0.001 ;$ dispersity $\left.=0.241, \mathrm{t}_{10}=4.879, \mathrm{p}<0.001\right)$. Intriguingly, dispersity reached a local minimum of around 0.1 at $50 \%$ THF, where uniform mLTSL10 was obtained. Thus, 50\% THF was deemed the optimal solvent condition to nanoprecipitate mLTSL10.

Once the formulation parameters were optimized, liposome size can be varied simply by changing the aqueous-to-organic volume ratio (VR) [18]. When VR was increased from 3 to 3.5, mLTSL10 size decreased from $194.4 \pm 28.2 \mathrm{~nm}$ (dispersity $=0.107 \pm 0.041, \mathrm{n}=8$ ) to 91.8 
$\pm 8.0 \mathrm{~nm}$ (dispersity $=0.091 \pm 0.033, \mathrm{n}=5)($ Supplementary Figure S3). The changes in size

was statistically significant $\left(\mathrm{t}_{14}=6.113, \mathrm{p}<0.001\right)$ but insignificant for dispersity $\left(\mathrm{t}_{14}=1.023\right.$, $\mathrm{p}>0.05)$. Representative correlograms and cumulant fits of the intensity correlation function were provided (Supplementary Figure S4). Further increase of VR to $4-6$ resulted in smaller $(<100 \mathrm{~nm})$ but more dispersed $(>0.2)$ mLTSL10. This could be attributed to higher membrane curvature of smaller liposomes, resulting in greater membrane distortion by SPION and heterogeneous populations [9,30]. In conclusion, uniformly distributed mLTSL10 can be prepared using 50\% THF, with two available sizes: $100 \mathrm{~nm}$ at VR of 3.5 and $200 \mathrm{~nm}$ at VR of 3. The zeta potential of the optimised mLTSL10 was $-7.83 \pm 0.806(n=6)$. Zeta potential of other conditions $(20-75 \% \mathrm{THF})$ ranges between -10 to $-7 \mathrm{mV}$, without major differences.

\subsection{SPION loading affects liposome dispersity without affecting membrane integrity}

260 In an attempt to maximize the SPION loading into mLTSL10, the organic phase (40 - 60\% THF) containing $10 \mathrm{mM}$ LTSL10 and 0 - $500 \mu \mathrm{g} \mathrm{mL} \mathrm{mL}^{-1}$ SPION (SPION-to-lipid ratio of 0 - 50, $\left.\mu \mathrm{g} \mu \mathrm{mol}^{-1}\right)$ was injected into the aqueous phase $\left(\left(\mathrm{NH}_{4}\right)_{2} \mathrm{SO}_{4}\right)$ at $60{ }^{\circ} \mathrm{C}$ with an aqueous-toorganic volume ratio (VR) of 3 and 3.5 (Figure 3). It was expected that as the loading of SPION exceeds a certain threshold limit, SPION and LTSL10 can no longer self-assemble uniformly,

265 larger and more dispersed samples would be formed.

Using the optimal solvent condition of 50\% THF, for large LTSL10 (VR of 3), SPION-to-lipid ratio can reach up to $50 \mu \mathrm{g} / \mu \mathrm{mol}$ with dispersity remaining $<0.2$. Interestingly, less amount of SPION (up to SPION-to-lipid ratio of $40 \mu \mathrm{g} / \mu \mathrm{mol}$ ) was tolerated by the small mLTSL10 (VR of 3.5), as the dispersity increased to 0.2. Determined by one-way ANOVA, SPION 270 concentration has statistically insignificant effect on mLTSL10 size for both large (VR of 3; $\left.\mathrm{F}_{2,11}=0.078, \mathrm{p}>0.05\right)$ and small mLTSL10 $\left(\mathrm{VR}\right.$ of $\left.3.5 ; \mathrm{F}_{4,20}=2.822, \mathrm{p}>0.05\right)$. On the other 
hand, while there was insignificant effect on larger mLTSL10 dispersity $\left(F_{2,11}=1.577, p>\right.$ 0.05), the effect on small mLTSL10 dispersity was significant $\left(F_{4,20}=4.563, p=0.009\right)$.

At 40\% THF where SPION are not well solubilized, mLTSL10 become dispersed as low as 275 SPION-to-lipid ratio of $15 \mu \mathrm{g} / \mu \mathrm{mol}$, affirming that self-assembly of mLTSL10 in $40 \%$ THF is unfavorable. On the other hand, despite greater solubility of SPION in 60\% THF, mLTSL10 have higher dispersity compared to those prepared in 50\% THF, which may be attributed to the unfavorable condition for formation of liposome per se in high concentration of THF. Considering the desirable size and dispersity of the resultant mLTSL10, the optimal conditions 280 for preparing mLTSL10 were using 50\% THF, with SPION-to-lipid ratio of $30 \mu \mathrm{g} / \mu \mathrm{mol}$ and VR of 3.5 .

The stability and membrane integrity of the optimized mLTSL10 was then characterized. Control experiments using only SPION without LTSL10 lipids were performed to validate the preparation processes. Initially, freshly prepared mLTSL10 after injection and annealing, were left overnight in ambient conditions without SEC purification; despite noticeable reduction in volume due to evaporation of THF, the solution remained clear and disperse (Figure 4a). In contrast, under the same conditions in the absence of LTSL10 lipids, the samples were unstable (Supplementary Figure S5a). SPION precipitated at the wall and the bottom of the container, which also agreed with the low solubility of SPION in the presence of $\left(\mathrm{NH}_{4}\right)_{2} \mathrm{SO}_{4}$. This 290 suggested that SPION have interacted with the LTSL10 lipids, presumably either embedded into the lipid bilayer or formed micelles, resulting in greater dispersion stability. To remove free SPION, freshly prepared mLTSL10 was purified by SEC. mLTSL10 passed through the column at the expected elute volume for liposomes and was collected as a clear, brownish solution (Figure $\mathbf{4 b}, \mathbf{4 c})$. In contrast, SPION alone could not be eluted and was trapped in the 295 frit at the top of the SEC column (Supplementary Figure S5b), which further suggested that SPION and LTSL10 liposomes eluted as one population. Then, SPION EE of purified 
mLTSL10 was characterized by AAS (Figure 4d). Large $200 \mathrm{~nm}$ mLTSL10 had a mean SPION EE of $85.85 \%$, significantly greater than small $100 \mathrm{~nm}$ mLTSL10 of $60.77 \%\left(\mathrm{t}_{10}=5.83\right.$, $\mathrm{p}<0.001)$. Liposomes of larger size have a lower surface curvature to accommodate SPION,

300 which may explain the higher loading capacity and thus lower dispersity of larger mLTSL10 prepared in 50\% THF $[9,30]$. Finally, to assess membrane integrity and drug loading capability, mLTSL10 were remotely loaded with DOX. As expected, DOX EE of all samples $(0-40$ $\mu \mathrm{g} / \mu \mathrm{mol}$ SPION-to-lipid ratio), were above $80 \%$ (Figure 4e). One-way ANOVA showed that the amount of SPION has no significant effect on the DOX EE $\left(\mathrm{F}_{3,9}=0.5386, \mathrm{p}>0.05\right)$.

\section{5 mLTSL10 Characterization}

The mean phase transition temperatures of LTSL10 and mLTSL10, defined as the onset temperature of the melting peak characterized by DSC, were $41.55{ }^{\circ} \mathrm{C}$ and $41.87{ }^{\circ} \mathrm{C}$ respectively, (Figure 5a, 5b). The increased transition peak height of mLTSL10, suggests that the fluid LTSL10 membranes were stabilized by the embedded SPION. Although the phase

310 transition temperature increased by $0.3{ }^{\circ} \mathrm{C}$, it remained within the range of mild hyperthermia $\left(40-42{ }^{\circ} \mathrm{C}\right)$ for thermosensitive release [2].

Representative TEM images showed that SPION have an iron core size of $4-5 \mathrm{~nm}$, in agreement with our previous characterization [22], which are smaller than the reported maximum SPION diameter of $6.5 \mathrm{~nm}$ for embedding into lipid bilayers (Figure 6a) [31].

315 Particles of similar size and morphology to SPION were observed in the TEM micrographs of mLTSL10 (Figure 6b). SPION were evenly distributed within the liposomes, which can be attributed to the two-dimensional projection and flattening of spherical liposomes, indicating the embedment of SPION into the LTSL10 lipid bilayer. Only a trivial quantity of individual SPION and no irregularly shaped agglomerate (as observed in free SPION) were observed, 
320 confirming the successful purification of unencapsulated and the effective embedment of SPION into LTSL10. 


\section{Discussion}

To date, majority of magnetoliposome have been prepared by batch methods in combination

with downsizing techniques of extrusion $[7,10,11,22]$ or sonication $[4,8,9,14]$. Indeed, due to the robustness of downsizing, there are little or no limitations on the quality of the preformed liposomes; large, heterogeneous, multilamellar vesicles can be processed into small, unilamellar vesicles. However, both techniques have limited scalability and come with their own disadvantages; sonication may degrade or contaminate the sample, while extrusion may suffer from high product losses [32]. In contrast, nanoprecipitation is a self-assembly process which homogeneous unilamellar liposomes are formed directly. There is no need for downsizing and homogenization, in turn the liposome quality is governed by the formulation and processing parameters.

To formulate thermosensitive magnetoliposome mLTSL10 via nanoprecipitation, the

335 formulation parameters must be first designed and optimized carefully to ensure all components can self-assemble into vesicles homogeneously. First, the thermosensitive formulation LTSL10 was selected, as we previously optimized for nanoprecipitation preparation [21]. Since the self-assembled liposomes are inevitably exposed to organic solvents during the nanoprecipitation, they will be prone to ethanol-induced interdigitation [33]. In contrast with typical LTSL formulations that contain about $4-5$ mol\% DSPE-PEG 2000 , DSPE$\mathrm{PEG}_{2000}$ was increased to $10 \mathrm{~mol} \%$ in LTSL10 to avoid the interdigitated gel phase [21].

Next, the solvent phases were optimized to ensure all components are well-solubilized and to prevent premature precipitation. Ammonium sulfate solution was selected as the aqueous phase to enable DOX remote loading; ethanol and THF were used to dissolve lipids and SPION, 345 respectively. THF has been an attractive organic solvent given its ability to dissolve hydrophobic substances while being water miscible. THF was commonly employed in the 
nanoprecipitation of inorganic and polymer nanoparticles [34-37]. Although SPION was metastable in $1 \%$ THF (in $99 \%$ ethanol), the presence of DSPE-PEG ${ }_{2000}$ rapidly disrupted the system. The relatively high solubility of DSPE-PEG 2000 in THF suggested that the surfacebounded THF could be displaced from the SPION, exposing them to ethanol resulting in precipitation. Conversely, DPPC and MSPC did not disrupt the SPION in $1 \%$ THF, in agreement with the relatively low solubility of DPPC and MSPC in THF. Consequently, the volume fraction of THF was inevitably increased to 50\% THF for a stable mLTSL10 mixture, where uniform mLTSL10 could be prepared. Similarly, others have used at least $40 \%$ THF in ethanol to prepare entrapped SPION in solid lipid nanoparticles [34] and conventional magnetoliposomes [35].

The effect of introducing THF in ethanol led to substantial increase in both size and dispersity for both LTSL10 and mLTSL10. Since self-assembly (via nanoprecipitation) is a spontaneous process that minimizes the molecular free energy, factors such as solubility and diffusivity of 360 individual components (lipids, SPION or solvents) in the system would all contribute to the resultant assembly's lowest energy state, hence the quality of the liposome population [16]. Others have observed that smaller polymeric nanoparticle sizes were obtained using solvents with greater diffusion coefficient in water [37,38]. The effect can be explained by the nanoprecipitation process, as solvents diffuse faster into water, solutes reach their critical 365 nucleation concentration earlier, providing less time for nuclei to grow in size, resulting in smaller nanoprecipitates [16]. Literature on the tertiary system of THF-ethanol-water were yet to be available; however, based on the data on the binary systems, the diffusion coefficient of ethanol-water is greater than that of THF-water $[39,40]$. Therefore, it may be reasoned that with increasing \% THF in ethanol, the diffusion coefficient would decrease, which could 370 explain our observation that larger mLTSL10 were formed as volume fraction of THF increased. 
Using lower concentration of THF is preferred; apart from forming dispersed mLTSL10 population, THF raises other concerns regarding scaling-up. High volatility and the ethereal smell of THF may be unpleasant for user to handle; moreover, THF is highly flammable and 375 can decompose into explosive peroxide, posing safety hazards. Furthermore, THF limits the choice of material for scale-up production. Microfluidics, the manipulation of fluid in microscale channels, is an emerging technique for nanoprecipitation of liposomes and acceleration of their clinical translation [20]. Major advantages of microfluidics in nanoparticles synthesis includes excellent manipulation of fluids, high reproducibility, tunable nanoparticle size and the process is readily scalable by continuous production and parallelization [19]. Currently, owing to the advancement in microfabrication, many microfluidic devices are polymer-based (such as polydimethylsiloxane and cyclic olefin copolymer) for their low-cost and ease of fabrication but have limited compatibility towards strong organic solvents like THF [34,41]. In fact, ongoing research has been conducted to advance material fabrication and microfluidic capabilities, in turn accelerating the development and translation of products prepared by nanoprecipitation $[41,42]$. In this regard, in order to translate the batch injection preparation of mLTSL10 to the continuous, scalable microfluidics method, upcoming microfluidic devices will have to tolerate a minimum of $50 \%$ THF in ethanol.

390 Thus far, there are only few reports on the nanoprecipitation of magnetoliposomes. Kulkarni et al. presented the nanoprecipitation of SPION-loaded solid lipid nanoparticles (SLNs), comprising abundant hydrophobic core lipids of triglycerides or sterols (up to $97.5 \mathrm{~mol} \%$ ) to entrap SPION in the SLN core [34]. Nevertheless, 40\% THF was still necessary to solubilize SPION in the initial lipid mixture. In comparison, Bixner and Reimhult used $100 \%$ THF to 395 prepare conventional (non-PEGylated) magnetoliposome [35]. Both reported formulations are based on unsaturated phospholipid, which have high fluidity and great tolerance towards 
interdigitation, thus suitable for preparation using nanoprecipitation. Although from the clinical translation point of view, unsaturated phospholipids are prone to oxidation and their low phase transition temperature can result in premature release at physiological temperature [43]. In 400 comparison, mLTSL10 comprises saturated phospholipids and PEG-lipid, are designed based on the most clinically advanced LTSL formulation, ThermoDox ${ }^{\circledR}$, which also offers triggered drug release capability. We carefully evaluated the effect of THF-ethanol binary solvent system on the mLTSL10 to attain the optimized condition of $50 \%$ THF, without the need of hydrophobic core lipids and avoided using excessive THF. Moreover, mLTSL10 was prepared 405 in ammonium sulfate which enabled the remote loading of hydrophilic cargo, such as DOX. mLTSL10 is capable of co-encapsulation of hydrophobic SPION and hydrophilic drugs, which is unprecedent in previous reports.

It is important to note that between different nanoprecipitation techniques (e.g. solvent injection, microfluidics, cross-flow injection), the mechanism of liposome formation remains

410 identical, thus the formulation parameters are readily transferable. However, processing parameters (e.g. volume ratio, mixing efficiency, flow rates) will be expected to vary according to the mixing conditions and efficiency of respective techniques [18]; as processing parameters of bench-scale preparation are also expected to differ from that of industrial scale [16]. Nonetheless, it remained promising and advantageous to prepare nanoparticles using 415 nanoprecipitation for its simplicity and capability of scale-up production for accelerating clinical translations. 


\section{Conclusion}

In summary, the presented results show that the nanoprecipitation technique successfully enabled the loading of SPION into the low temperature-sensitive liposomal system, yielding spherical, nano-sized homogeneous magneto-thermosensitive liposomes, mLTSL10. The effect of the formulation parameters, solvent system and SPION concentration, were investigated and optimized. Minimal amount of 50\% THF was required to yield uniform and stable mLTSL10. Thermosensitivity and membrane integrity were not affected by the inclusion of SPION. The nanoprecipitation technique allows efficient SPION loading into liposomes and eliminates the need for post processing such as extrusion or sonication. It is envisaged that in combination with scalable nanoprecipitation platform such as microfluidics, the development of mLTSL10 as a responsive drug delivery carrier will be further accelerated.

\section{Conflicts of interest}

430 There are no conflicts to declare.

\section{Acknowledgment}

This work was supported by the Prostate Cancer UK (Grant CDF12-002), and the Engineering and Physical Sciences Research Council (EPSRC) (EP/M008657/1). 


\section{References}

[1] G.Kong, G.Anyarambhatla, W.P.Petros, R.D.Braun, O.M.Colvin, D.Needham, M.W.Dewhirst, Efficacy of liposomes and hyperthermia in a human tumor xenograft model: importance of triggered drug release., Cancer Res. 60 (2000) 6950-7.

[2] D.Needham, G.Anyarambhatla, G.Kong, M.W.Dewhirst, A new temperature-sensitive liposome for use with mild hyperthermia: Characterization and testing in a human tumor xenograft model, Cancer Res. 60 (2000) 1197-1201.

[3] Y.Dou, K.Hynynen, C.Allen, To heat or not to heat: Challenges with clinical translation of thermosensitive liposomes, J. Control. Release. 249 (2017) 63-73.

[4] Z.Al-Ahmady, N.Lozano, K.C.Mei, W.T.Al-Jamal, K.Kostarelos, Engineering thermosensitive liposome-nanoparticle hybrids loaded with doxorubicin for heattriggered drug release, Int. J. Pharm. 514 (2016) 133-141.

[5] N.Forbes, A.Pallaoro, N.O.Reich, J.A.Zasadzinski, Rapid, Reversible Release from Thermosensitive Liposomes Triggered by Near-Infra-Red Light, Part. Part. Syst. Charact. 31 (2014) 1158-1167.

450 [6] N.Forbes, J.E.Shin, M.Ogunyankin, J.A.Zasadzinski, Inside-outside self-assembly of light-activated fast-release liposomes, Phys. Chem. Chem. Phys. 17 (2015) 1556915578.

[7] J.-P.Fortin-Ripoche, M.S.Martina, F.Gazeau, C.Ménager, C.Wilhelm, J.-C.Bacri, S.Lesieur, O.Clément, Magnetic Targeting of Magnetoliposomes to Solid Tumors with MR Imaging Monitoring in Mice: Feasibility, Radiology. 239 (2006) 415-424.

[8] G.Béalle, R.DiCorato, J.Kolosnjaj-Tabi, V.Dupuis, O.Clément, F.Gazeau, C.Wilhelm, C.Ménager, Ultra magnetic liposomes for MR imaging, targeting, and hyperthermia, 
Langmuir. 28 (2012) 11834-11842.

[9] R.Martínez-González, J.Estelrich, M.A.Busquets, Liposomes loaded with hydrophobic 460 iron oxide nanoparticles: Suitable T2 contrast agents for MRI, Int. J. Mol. Sci. 17 (2016) $1-14$.

[10] E.Amstad, J.Kohlbrecher, E.Müller, T.Schweizer, M.Textor, E.Reimhult, Triggered release from liposomes through magnetic actuation of iron oxide nanoparticle containing membranes, Nano Lett. 11 (2011) 1664-1670.

465 [11] Y.Guo, Y.Zhang, J.Ma, Q.Li, Y.Li, X.Zhou, D.Zhao, H.Song, Q.Chen, X.Zhu, Light/magnetic hyperthermia triggered drug released from multi-functional thermosensitive magnetoliposomes for precise cancer synergetic theranostics, J. Control. Release. 272 (2018) 145-158.

[12] A.C.Silva, T.R.Oliveira, J.B.Mamani, S.M.F.Malheiros, L.Malavolta, L.F.Pavon, T.T.Sibov, E.Amaro, A.Tannús, E.L.G.Vidoto, M.J.Martins, R.S.Santos, L.F.Gamarra, Application of hyperthermia induced by superparamagnetic iron oxide nanoparticles in glioma treatment., Int. J. Nanomedicine. 6 (2011) 591-603.

[13] B.Le, M.Shinkai, T.Kitade, H.Honda, J.Yoshida, T.Wakabayashi, T.Kobayashi, Preparation of tumor-specific magnetoliposomes and their application for hyperthermia, J. Chem. Eng. Japan. 34 (2001) 66-72.

[14] Y.Chen, A.Bose, G.D.Bothun, Controlled Release from Bilayer- Decorated Magnetoliposomes via Electromagnetic Heating, ACS Nano. 4 (2010) 3215-3221.

[15] S.Hua, M.B.C.deMatos, J.M.Metselaar, G.Storm, Current Trends and Challenges in the Clinical Translation of Nanoparticulate Nanomedicines: Pathways for Translational Development and Commercialization, Front. Pharmacol. 9:790 (2018). 
[16] C.J.Martínez Rivas, M.Tarhini, W.Badri, K.Miladi, H.Greige-Gerges, Q.A.Nazari, S.A.Galindo Rodríguez, R.Á.Román, H.Fessi, A.Elaissari, Nanoprecipitation process: From encapsulation to drug delivery, Int. J. Pharm. 532 (2017) 66-81.

[17] L.Capretto, D.Carugo, S.Mazzitelli, C.Nastruzzi, X.Zhang, Microfluidic and lab-on-achip preparation routes for organic nanoparticles and vesicular systems for nanomedicine applications, Adv. Drug Deliv. Rev. 65 (2013) 1496-1532.

[18] C.C.L.Cheung, W.T.Al-Jamal, Sterically stabilized liposomes production using staggered herringbone micromixer: Effect of lipid composition and PEG-lipid content, Int. J. Pharm. 566 (2019) 687-696.

[19] J.Ma, S.M.Y.Lee, C.Yi, C.W.Li, Controllable synthesis of functional nanoparticles by microfluidic platforms for biomedical applications-a review, Lab Chip. 17 (2017) 209226.

[20] P.M.Valencia, O.C.Farokhzad, R.Karnik, R.Langer, Microfluidic technologies for accelerating the clinical translation of nanoparticles, Nat. Nanotechnol. 7 (2012) 623629.

[21] C.C.L.Cheung, G.Ma, A.Ruiz, W.T.Al-Jamal, Microfluidic Production of LysolipidContaining Temperature-Sensitive Liposomes, J. Vis. Exp. 157 (2020) e60907.

[22] N.Kostevšek, C.C.L.Cheung, I.Serša, M.E.Kreft, I.Monaco, M.Comes Franchini, J.Vidmar, W.T.Al-Jamal, Magneto-Liposomes as MRI Contrast Agents: A Systematic Study of Different Liposomal Formulations, Nanomaterials. 10 (2020) 889.

[23] X.Teng, H.Yang, Effects of surfactants and synthetic conditions on the sizes and selfassembly of monodisperse iron oxide nanoparticles, J. Mater. Chem. 14 (2004) 774779. 
[24] T.P.Niebel, F.J.Heiligtag, J.Kind, M.Zanini, A.Lauria, M.Niederberger, A.R.Studart, Multifunctional microparticles with uniform magnetic coatings and tunable surface chemistry, RSC Adv. 4 (2014) 62483-62491.

[25] R.F.T.Stepto, Dispersity in polymer science (IUPAC Recommendations 2009), Pure Appl. Chem. 81 (2009) 351-353.

[26] O.López, A.DeLa Maza, L.Coderch, C.López-Iglesias, E.Wehrli, J.L.Parra, Direct formation of mixed micelles in the solubilization of phospholipid liposomes by Triton X-100, FEBS Lett. 426 (1998) 314-318.

[27] K.J.Rothman, No adjustments are needed for multiple comparisons., Epidemiology. 1 (1990) 43-6.

[28] D.J.Saville, Multiple Comparison Procedures: The Practical Solution, Am. Stat. 44 (1990) 174.

[29] M.A.Obeid, I.Khadra, A.B.Mullen, R.J.Tate, V.A.Ferro, The effects of hydration media on the characteristics of non-ionic surfactant vesicles (NISV) prepared by microfluidics, Int. J. Pharm. 516 (2017) 52-60.

[30] C.Bonnaud, C.A.Monnier, D.Demurtas, C.Jud, D.Vanhecke, X.Montet, R.Hovius, M.Lattuada, B.Rothen-Rutishauser, A.Petri-Fink, Insertion of nanoparticle clusters into vesicle bilayers, ACS Nano. 8 (2014) 3451-3460.

[31] H.S.Wi, K.Lee, H.Kyu Pak, Interfacial energy consideration in the organization of a quantum dot-lipid mixed system, J. Phys. Condens. Matter. 20 (2008) 494211.

[32] A.Wagner, K.Vorauer-Uhl, Liposome Technology for Industrial Purposes, J. Drug Deliv. 2011 (2011) 1-9.

[33] M.Patra, E.Salonen, E.Terama, I.Vattulainen, R.Faller, B.W.Lee, J.Holopainen, 
M.Karttunen, Under the influence of alcohol: The effect of ethanol and methanol on lipid bilayers, Biophys. J. 90 (2006) 1121-1135.

[34] J.A.Kulkarni, Y.Y.C.Tam, S.Chen, Y.K.Tam, J.Zaifman, P.R.Cullis, S.Biswas, Rapid synthesis of lipid nanoparticles containing hydrophobic inorganic nanoparticles, Nanoscale. 9 (2017) 13600-13609.

[35] O.Bixner, E.Reimhult, Controlled magnetosomes: Embedding of magnetic nanoparticles into membranes of monodisperse lipid vesicles, J. Colloid Interface Sci. 466 (2016) 62-71.

[36] P.M.Valencia, P.A.Basto, L.Zhang, M.Rhee, R.Langer, O.C.Farokhzad, R.Karnik, Single-step assembly of homogenous lipid-polymeric and lipid-quantum dot nanoparticles enabled by microfluidic rapid mixing, ACS Nano. 4 (2010) 1671-1679.

[37] W.Huang, C.Zhang, Tuning the Size of Poly(lactic-co-glycolic Acid) (PLGA) Nanoparticles Fabricated by Nanoprecipitation, Biotechnol. J. 13 (2018) 1700203.

[38] P.Legrand, S.Lesieur, A.Bochot, R.Gref, W.Raatjes, G.Barratt, C.Vauthier, Influence of polymer behaviour in organic solution on the production of polylactide nanoparticles by nanoprecipitation, Int. J. Pharm. 344 (2007) 33-43.

[39] D.G.Leaist, K.MacEwan, A.Stefan, M.Zamari, Binary mutual diffusion coefficients of aqueous cyclic ethers at $25^{\circ} \mathrm{C}$. Tetrahydrofuran, 1,3-dioxolane, 1,4-dioxane, 1,3dioxane, tetrahydropyran, and trioxane, J. Chem. Eng. Data. 45 (2000) 815-818.

[40] S.Parez, G.Guevara-Carrion, H.Hasse, J.Vrabec, Mutual diffusion in the ternary mixture of water + methanol + ethanol and its binary subsystems, Phys. Chem. Chem. Phys. 15 (2013) 3985.

[41] X.Hou, Y.S.Zhang, G.T.Santiago, M.M.Alvarez, J.Ribas, S.J.Jonas, P.S.Weiss, 
A.M.Andrews, J.Aizenberg, A.Khademhosseini, Interplay between materials and microfluidics, Nat. Rev. Mater. 2 (2017) 17016.

[42] S.Goyal, A.E.Economou, T.Papadopoulos, E.M.Horstman, G.G.Z.Zhang, Y.Gong, P.J.A.Kenis, Solvent compatible microfluidic platforms for pharmaceutical solid form screening, RSC Adv. 6 (2016) 13286-13296.

555 [43] M.K.Yeh, H.I.Chang, Clinical development of liposome based drugs: formulation, characterization, and therapeutic efficacy, Int. J. Nanomedicine. 7 (2011) 49. 


\section{Figures}

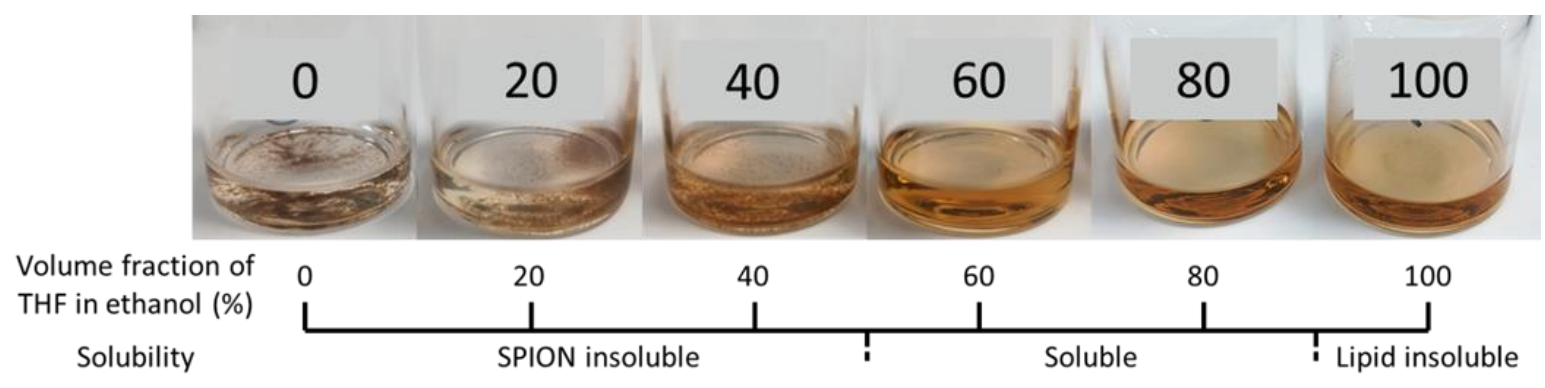

560 Figure 1. Solubility of SPION and LTSL10 lipid in THF/ethanol binary solvent mixtures.

Photos of $200 \mu \mathrm{g} \mathrm{mL}^{-1}$ SPION with $10 \mathrm{mM} \mathrm{LTSL10}$ dispersed in ethanol with (left to right) 0 , $20,40,60,80,100 \% \mathrm{THF}$ at $20{ }^{\circ} \mathrm{C}$. 

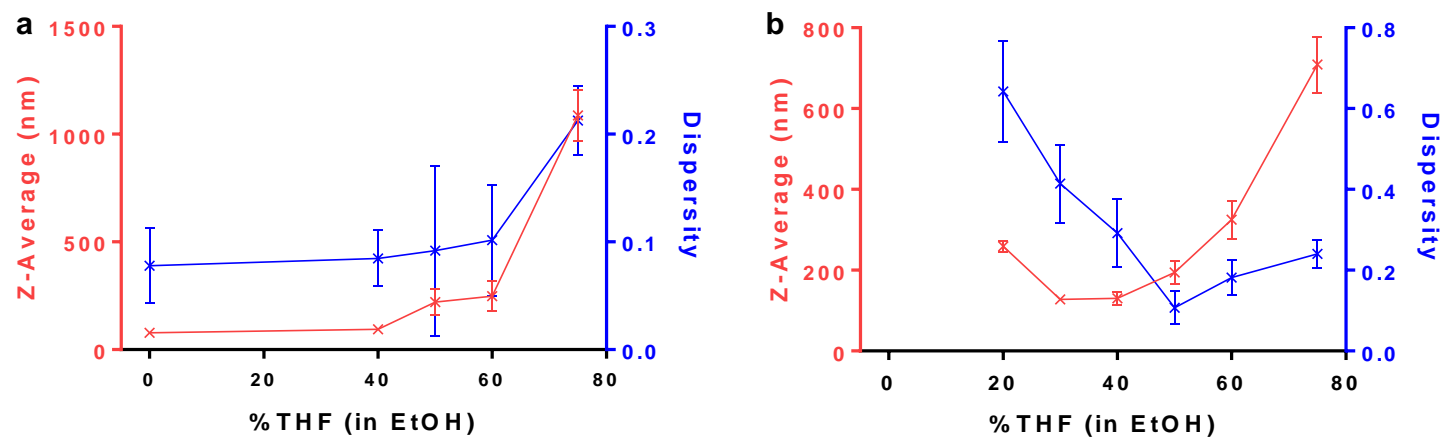

565 Figure 2. Effect of THF volume fraction on LTSL10 and mLTSL10 size and dispersity.

(a) $10 \mathrm{mM}$ LTSL10 and (b) $10 \mathrm{mM}$ LTSL10 containing $300 \mu \mathrm{g} \mathrm{mL}^{-1}$ SPION, dispersed in $0-$ $75 \%$ THF, were injected into $\left(\mathrm{NH}_{4}\right)_{2} \mathrm{SO}_{4}$ with volume ratio of 3 . Data are mean $\pm \mathrm{SD}(\mathrm{n}=2-$ 7). 

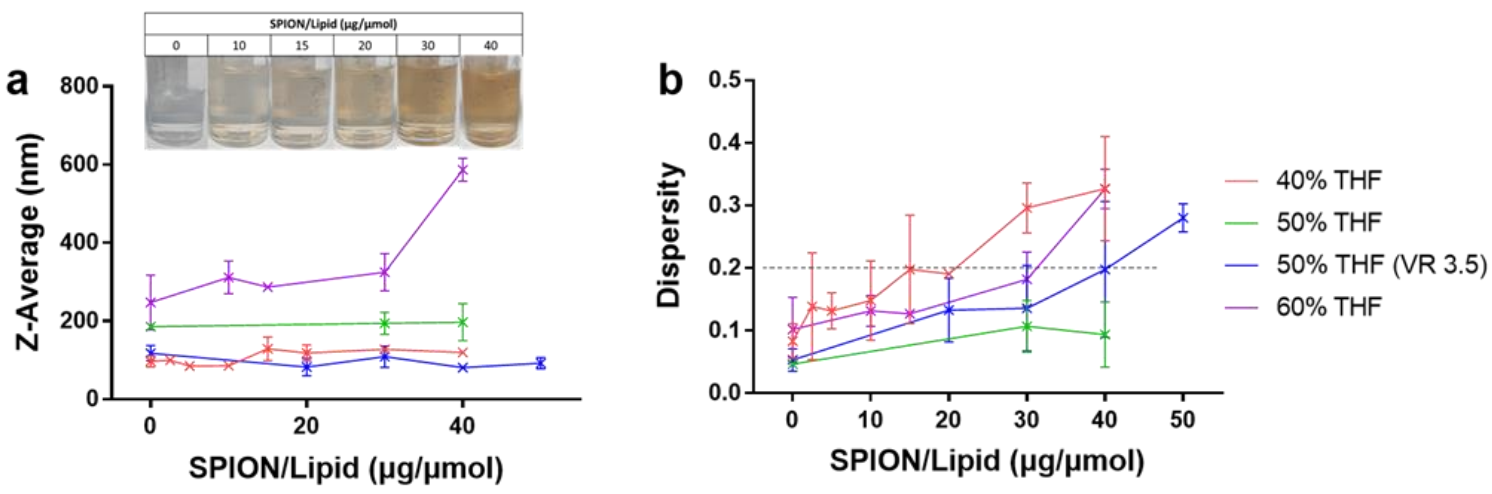

570

Figure 3. Effect of SPION concentration and \% THF on mLTSL10 size and dispersity. (a)

Z-average diameter and (b) dispersity of mLTSL10 with SPION-to-lipid ratio of $0-50$ $(\mu \mathrm{g} / \mu \mathrm{mol})$, dispersed in $40-60 \% \mathrm{THF}$, were injected into (NH4)2SO4 with a volume ratio of 3 (or 3.5). Data are mean \pm SD $(n=2-7)$. Inset are representative photo of mLTSL10 with 575 SPION-to-lipid ratio of $0-40(\mu \mathrm{g} / \mu \mathrm{mol})$ 

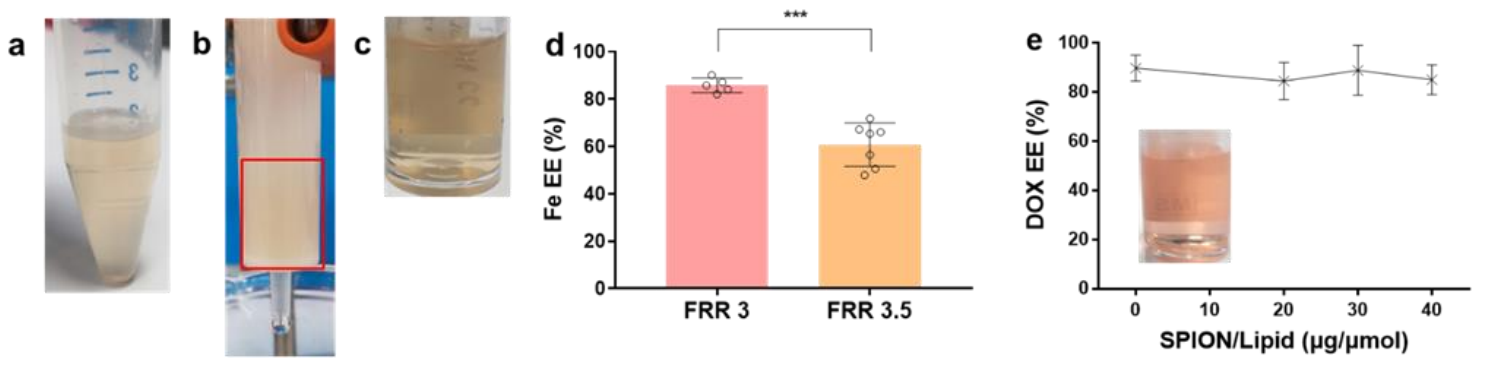

Figure 4. mLTSL10 preparation and characterization (a-c) mLTSL10 loaded with SPIONto-lipid ratio of $30(\mu \mathrm{g} / \mu \mathrm{mol})$, dispersed in 50\% THF, injected with a volume ratio of 3.5. (a) mLTSL10 remained disperse at $20{ }^{\circ} \mathrm{C}$ overnight without purification. (b) mLTSL10 passing 580 through SEC column during purification. Red box indicates the location of the mLTSL10. (c) Representative photo of collected mLTSL10 after SEC purification. (d) SPION encapsulation efficiency of mLTSL10 prepared in 50\% THF with injection volume ratio of 3 and 3.5. $(n=4$ -7). Two-tailed unpaired $t$-test; ***, $\mathrm{p}<0.001$. (e) DOX encapsulation efficiency of mLTSL10 dispersed in $50 \%$ THF with injection volume ratio of $3.5(n=2-7)$. Inset is representative 585 photo of purified mLTSL10 after DOX remote loading. Data are mean \pm SD. 

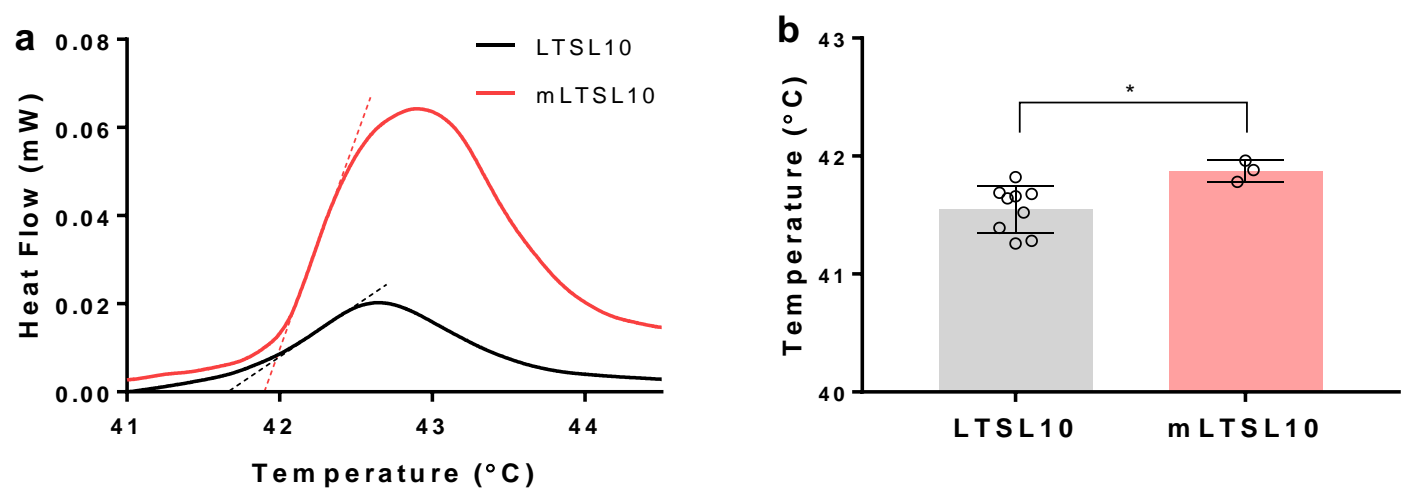

Figure 5. Thermal analysis of LTSL10 and mLTSL10. (a) Thermographs and (b) Onset phase transition temperature of LTSL10 and mLTSL10 characterized by DSC $(n=3-9)$.

590 Dotted lines as tangent of the point of maximum slope, are added as a visual aid of the onset phase transition temperature ( $\mathrm{x}$-intercept of the tangent line). Two-tailed unpaired $t$-test; ${ }^{*}, \mathrm{p}<$ 0.05. Data are mean \pm SD. 

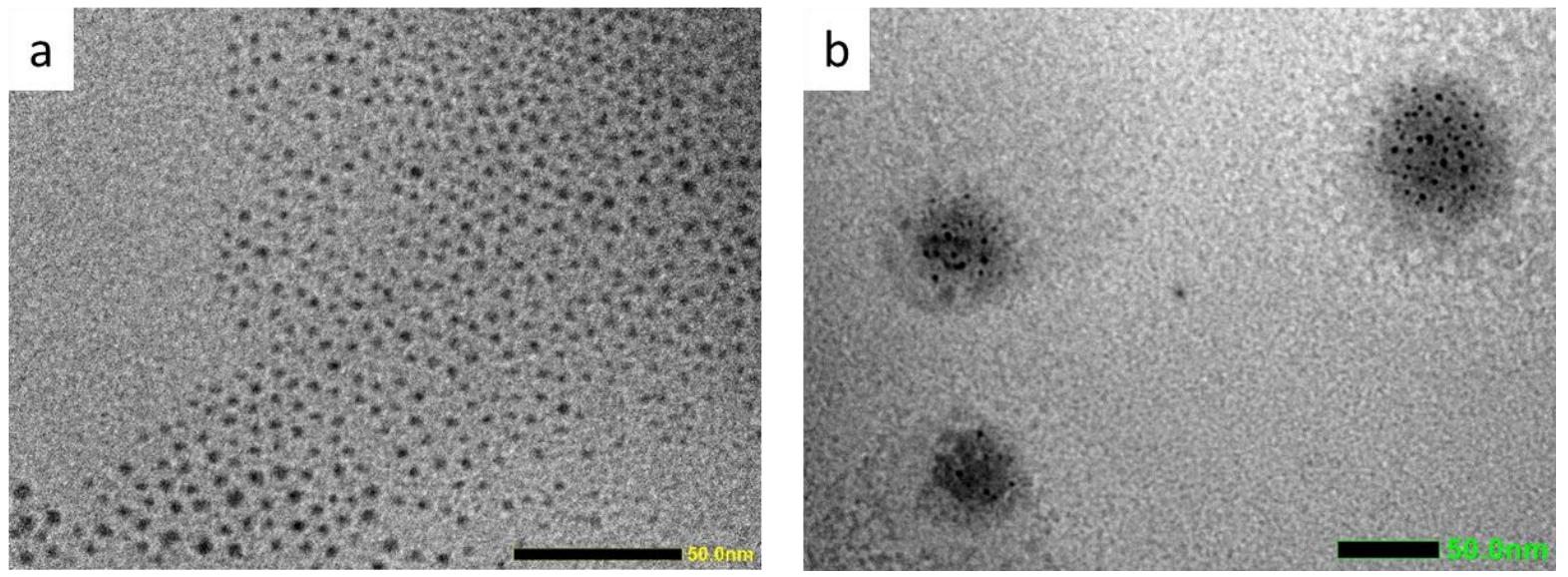

595 Figure 6. TEM micrographs of (a) SPION in THF, and (b) optimized mLTSL10 (dispersed in $50 \% \mathrm{THF}$, injection volume ratio of 3.5). Scale bars are $50 \mathrm{~nm}$. 